\title{
XIX.
}

\section{Untersuchungen zur Ephemeris des Diktys von Kreta.}

(Fortsetzung.)

Mehr noch als bei dem allgemeinen Erzählungsgang ist diese Diskrepanz von Kedren und Septimius innerhalb der einzelnen Nummern selbst sichtbar. So z. B. gehören in der Darstellung des Palladionraubes Malalas und Kedren entschieden enger zusammen als Kedren und Septimius. Patzig hat dargethan, dafs die Erzählung des Kedrenos dadurch entstanden sei, daßs er die disiecta membra des Malalas wieder zusammengelesen und vereinigt hat ${ }^{64}$ ). Auch IA stimmt hier, wie wir schon oben gesehen, ziemlich genau mit Kedren, gehört aber im Hinblick auf die Abfolge der Erzählungsmomente (1. Orakel über das Palladium 2. Geschichte des P. 3. Raub des P.) nochmals enger mit Septimius zusammen ${ }^{65}$ ). Auch das Folgende ist nicht minder ungünstig für Noacks Kedrenhypothese. Malalas fügt an den Bericht über den Friedenschlufs und den Empfang der den Troianern auferlegten Kontribution $(113,4)$ die Erzählung, Odysseus habe die Griechen, welche sich zur Heimfahrt anschickten, bei den Opfern beschworen, von diesem Vorhaben abzustehen, bis man es mit dem hölzernen Pferd probiert habe. So auch - z. T. verbo tenus vgl. die Uebergangsformel 'I $\lambda$ iou, $\pi$ piv . . . - Kedren 230, 17-20. Septimius aber weif nichts von einem Opfer, nichts von dem be-

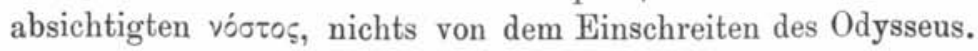
$\mathrm{Er}$ hat vielmehr eine andere Anordnung. Nach dem Friedenschlufs und der Erwähnung der Kriegsentschädigung (= Mal

64) Byz. Ztschr. II 428.

$\left.{ }^{65}\right)$ Insbesondere stimmt IA noch besonders mit Septimius darin, daß beide den Raub des Götterbildes vollführt werden lassen gelegentlich der Gesandtschaft des Diomedes und Odysseus (Sept. V 4; IA ed. Heinr. 10, $22 \mathrm{vgl}$. Suidas s. v. Il $\alpha \lambda \lambda . \dot{\delta} \delta$ :ov). 
$=$ Kedren = IA) kommt bei ihm a) der Beschluf, der Göttin Minerva ein Geschenk zu weihen $(93,20)$ sowie die Prophezie des Helenus $(93,21)$; b) die Gesandtschaft der firmatores pacis (V 10). - Bei Malalas folgt nun die Erzählung von dem hölzernen Pferd und dem damit zusammenhängenden Verderben Troias, eine Stelle, die unter allen von Noack angeführten Momenten fast allein einige Schwierigkeit bereitet. Kedrenos 231, 4 bietet die Erzählung von Sinon und seiner Täuschung der Troer. Woher hat Kedren diesen Zug? Malalas ist er fremd und auch im vollständigen Malalas ist wohl nie etwas davon gestanden, wie wir aus der genauen Uebereinstimmung mit der Ekloge (207, 15 sqq.) folgern müssen. Auch bei IA suchen wir vergebens nach ihm (IA ed. Heinr. 10, 7). Zwar ist die Stelle im cod. Vindob. 99 offenbar nur sehr auszüglich erhalten $(10,7)$. Aber hier tritt ein von IA abhängiger Byzantiner ergänzend ein, Manasses ${ }^{66}$ ), der v. 1421 sqq. die Geschichte des hölzernen Pferdes sehr breit und in einer die Sinonepisode ausschliefienden Weise ${ }^{67}$ ) erzählt. Man könnte einigermafen versucht sein, den fraglichen Bestandteil der Kedren'schen Diktyserzählung mit Noack auf eine hinter Malalas zurückliegende Chronik zurückzuführen. Denn E. Bethe hat im Rhein. Mus. 46 S. 519 die Sinongeschichte als ein altes Motiv erwiesen, das also nicht eine Erfindung der Byzantiner zu sein braucht und an sich dem Diktysbuche recht gut zugeeignet werden könnte ${ }^{68}$ ). Allein die Darstellung des Septimius ist ebenfalls derart, dafs sie den Rat des Sinon, das Pferd in die Stadt zu ziehen, ausschlieks ${ }^{69}$ ). Bei ihm giebt Sinon nur das Feuerzeichen (V 12), wovon bei Kedren nicht die, Rede ist. Ja Kedren nennt nicht einmal ausdrücklich den

$\left.{ }^{66}\right)$ Vgl. Patzig Byz. Ztschr. I 139; Programm 1892 S. 5. 12. 17. Byz. Ztschr. II 414. Bestätigt wird diese Auffassung durch den Wiener Text ed. Heinr., der z. B. den Traum der Hekuba und die Datierung des troianischen Kriegs unter David mit Manasses $(1120-1127)$ gemein hat.

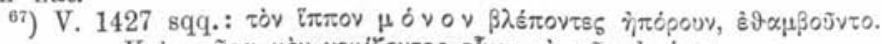

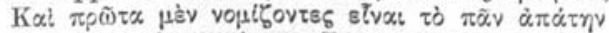

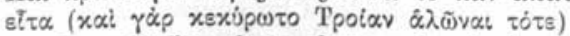

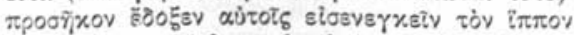

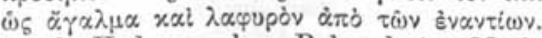

$\left.{ }^{68}\right)$ Genau wie Kedren schon Palaephatus Mythogr. gr. Westerm. p. 283,7 .

$\left.{ }^{69}\right) \mathrm{V} 11$. 


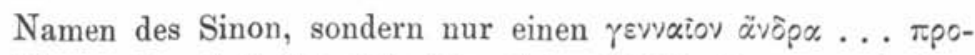

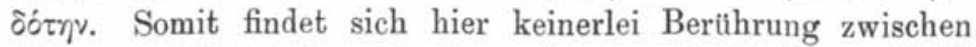
Kedren und Septimius. Auch sonst gehen sie in diesem Stück auseinander. Bei Kedren steigen die im Bauche des Pferdes Verborgenen nachts heraus und töten den Priamus und toे

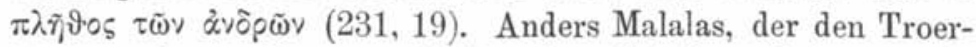
könig erst von den zurückkehrenden Griechen niedergemacht werden läft $(113,10-11)$; ähnlich wie Mal. auch Septimius p. 97,8 und IA p. 10,12 . Auch einen andern Gesichtspunkt möchte ich nicht unerwähnt lassen. Bei Septimius haben es die Griechen mit ihrer Kriegslist unverkennbar darauf abgesehen, nicht so fast die bewaffneten Helden in die feindliche Stadt einzuschmuggeln, als vielmehr die Troianer zu verlocken, eine möglichst große Bresche in die Mauer zu legen (vgl. V 11 u. 12). Eine ziemlich übereinstimmende Betonung desselben Momentes findet sich bei Malalas, wo von dem nächtlichen Handstreich der griechischen Helden in Troia keine Silbe zu lesen ist. In demselben Sinne, nur noch etwas deutlicher erzählt IA, während dagegen Kedrenos 231, 16 gerade den Vorstok der versteckten Griechen mit allem Nach-

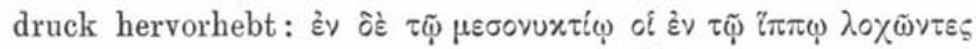

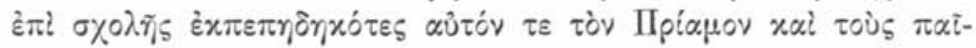

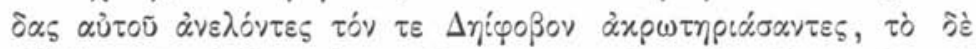

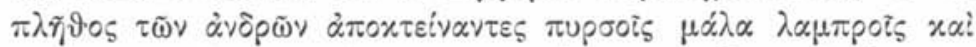

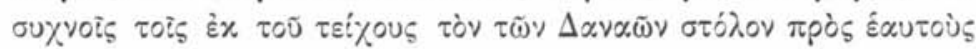

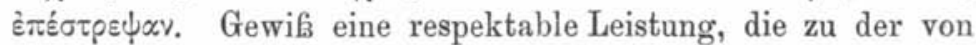
IA 10,6 (=Verg. Aen. II 261) auf 9 Helden bezifferten Mannschaft im umgekehrten Verhältnis steht. Und sehen wir genauer zu, so hat Kedrenos in der That diese Zahl auf das nahezu Dreifache erhöht, wie ich glauben möchte, um das schreiende Mifverhältnis zu mildern. Bei ihm steigen nicht 9, sondern 24 Helden in das Innere des Rosses ${ }^{69}$ ).

69a) Warum gerade 24? Ist diese Zahl vielleicht veranlaßt durch das š $\mu \varepsilon \sigma o v v x \tau i \varphi \varphi=2 \times 12$ ? Der symbolische Wert der Zahl 12 ist ja bekannt genug. Vgl. A. Wirth, Chronographische Späne Frankf. a. M. 1894 S. 39 f. Möglich auch, daß die 4 wohl gleichzeitig und getrennt ausgeführt gedachten Aktionen der Helden in Troja diese künstliche Zahl nahelegten $=4 \times 6$; ein Analogon bietet Chrysologus Rede no. 170 bei Ficker DA S. 5, wo die Zahl 12 aufgelöst ist in $3 \times 4$ (vgl. noch H. Usener, Das Weihnachtsfest S. 20; Texte und Unter- 
Fassen wir nun das bisherige Resultat kurz zusammen, so ist zuvörderst zu konstatieren: Sämtliche Diktysberichte stimmen in einem Grade überein, dafs eine gemeinsame, in letzter Instanz allen zu Grunde liegende Quelle deutlich erkennbar ist. Wo aber Kedrenos seine eigenen Wege geht, trifft er durchweg nicht mit Septimius zusammen, sondern mit den andern Byzantinern, die ihrerseits wieder häufig genug über Kedrenos hinaus mit Septimius übereinkommen. Damit fällt aber der von Noack behauptete höhere Grad der Verwandtschaft des Kedrenos mit Septimius bezw. dem griechischen Diktys dahin.

In Konsequenz seiner Quellenhypothese war Noack gezwungen, den Kedrenschen Troiabericht als ein einheitliches Ganzes auszugeben. Von einer Kompilation könne nicht die Rede sein (S. 423). Diese Ansicht wird sich schwer aufrecht halten lassen. Dafi Kedren aus Malalas geschöpft haben muf, glaube ich oben dargethan zu haben. Die Rechnung geht jedoch nicht auf: es bleibt ein Rest, für den wir mindestens noch eine Quelle fordern müssen. Damit fällt die Voraussetzung der angeblichen Einheitlichkeit der Kedren'schen Troika. Uebrigens ist ja an einer Stelle der Quellenwechsel deutlich in-

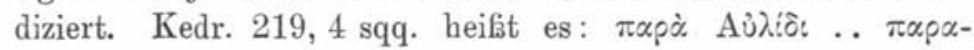

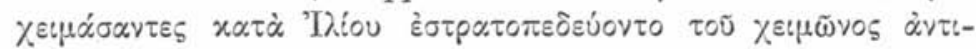

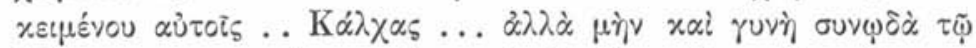

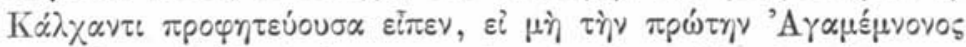

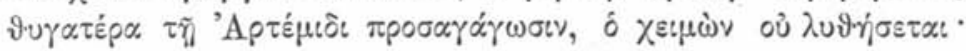

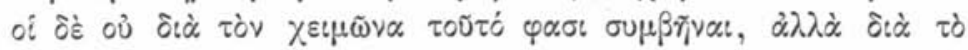

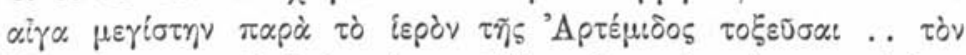

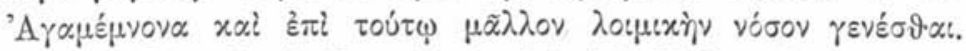
Mit der Formel of ò scheinlich einer neuen Vorlage zu. Noack hat an dieser Gegeninstanz nicht vorbeizukommen vermocht. Er giebt zu, eine solche Sonderung zweier Berichte wolle schlecht in die Darstellung des Augenzeugen Diktys passen. Nur sei nicht zu

suchungen hrsg. v. Gebhard und Harnack VWI (1892) S. 447). Eine solche allerdings mehr als puerile Zahlensymbolik dürfen wir einem Byzantiner vom Schlage des Kedren ohne weiteres zutrauen, wenn gleich diese Dinge in jenes Gebiet der byzantinischen Litteratur gehören, das noch sehr mangelhaft erforscht ist (cfr. Krumbacher Byz. Littg. ${ }^{2}$ S. 630). 
übersehen, daß3 auch Septimius an dieser Stelle zwei Möglichkeiten eingeführt habe. II 19 sagt er nämlich: neque multo post irane caelesti an ob mutationem aeris .. lues invadit. In Wirklichkeit ist die Septimiusstelle der des Kedrenos gar nicht parallel: dort eine bloße rationalistische Disjunktion, hier die formelle Einführung einer neuen Ueberlieferung; dort handelt es sich um die Frage, wodurch speciell die Pest entstanden sei, hier, wodurch überhaupt die Abfahrt der Griechen verzögert worden. Also der Hinweis auf Septimius geht $\mathrm{m}$. E. daneben. Doch angenommen, die Doppelversion des Kedrenos sei im griechischen Diktys bereits grundgelegt gewesen, aber erst Kedren habe die Wendung of $\delta \dot{\varepsilon} \varphi \propto \sigma$ e eingefügt und dadurch den Schein einer neuen, von der bisherigen verschiedenen Quelle erweckt, wie sollen wir uns denn dort diese Verbindung der beiden Versionen denken, durch die der Eindruck einer mythographisierenden Aufzählung ${ }^{70}$ ) ferngehalten wurde? Ein solcher ist aber aus den Memoiren des Diktys schlechterdings auszuschliefen. Als eine rationalistische Improvisation im Sinne der eben genannten SDiktysstelle kann die erste Version des Kedren ohnehin nicht angesehen werden. Denn sie ist ein Motiv der klassischen Sagenpoesie und bereits bei Antonius Liberalis ${ }^{71}$ ) in der Kedrenschen Fassung verwertet. Also von den beiden Versionen hat nur eine im griechischen Diktys gestanden und das ist natürlich keine andere als jene, welche auch Septimius bietet. Sie bat Kedren zu seiner ersten, aus Malalas 98, 6 sqq. entnommenen Version mit der Verbindungsformel oi $\delta \dot{\varepsilon} \varphi \propto \sigma:$ hinzugefügt, vermutlich aus IA. Daß er diesen überhaupt benützt hat, zeigt der Grazer IAtext. Kedren 216, 11 setzt den trojanischen Krieg in die Epoche Saul-David offenbar in sklavischer Anlehnung an seine Quelle. Denn 149, 5 verlegt er ihn in die Zeit des Eli. Auffallenderweise finden wir denselben Widerspruch auch bei IA. Er

$\left.{ }^{70}\right)$ Bei den Mythographen werden die verschiedenen Sagen in derselben Weise und mit derselben Wendung registriert, wie wir sie bei Kedren treffen, vgl. Westermann, Mythogr. gr. p. 76,$8 ; 77,19 ; 189$, $12 ; 189,15 ; 192,16 ; 273,9$ u. s. w. Man könnte auch an die Schreibweise der Paradoxographen erinnern, vgl. Paradoxograph. gr. ed. Westerm. pag. 6 , XIV; 7, XIX; 19, LXV u. passim.

71) Westermann l. c. p. 226,8 ; Fuchs, de varietate fabularum Troicarum quaestiones p. 93. 
läft 7, 17 ed. Heinr. den Priam bei David um Hilfstruppen bitten. Unter dessen Regierung spielt also der Krieg (vgl.

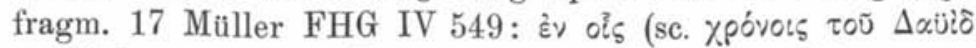

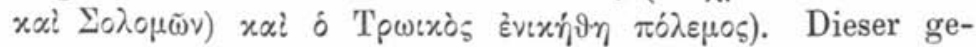
meinsame Widerspruch zeigt $\mathrm{m}$. E. deutlicher als alles Uebrige die Abhängigkeit des Kedrenos von IA.

Aus Malalas und Johannes Antiochenus ist demnach die Troiaerzählung des Kedrenos kombiniert ${ }^{72}$ ). Eine dritte Quelle,

$\left.{ }^{72}\right)$ Auf IA werden wir speciell ff. Stücke zurückführen müssen: die Chryseisepisode 222,7 ; die listige Herbeischaffung der Iphigenie 219,16 ; die mit der Opferung der Iphigenie zusammenhängenden Vorgänge wenigstens teilweise; die Geschichte des Palamedes mitten in den Vorgängen in Aulis (Kedr. 220, 4 vgl. Manasses 1330); die Angabe

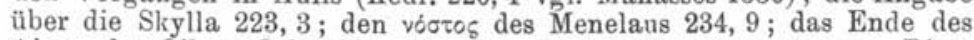

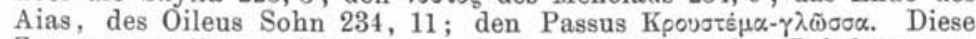
Zusammenstellung will übrigens nicht vollständig sein. Bei der engen Verwandtschaft des Malalas und IA ist oft schwer zu entscheiden, welche Stücke aus Malalas und welche aus IA stammen. Sicher aus Malalas ist z. B. die Bemerkung Kedr. 218, 17 (vgl. Gleye, Byz. Ztschr. V 454). Ebenso ist die umfangreiche Notiz Kedr. 223, 4-14 ein Ein-

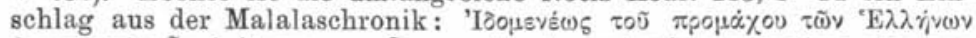

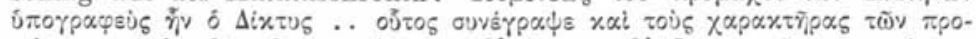

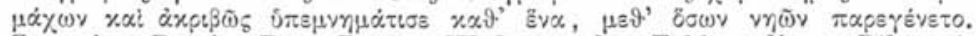
Zwar hat Patzig (Byz. Ztschr. IV S. 27) das Fehlen dieses Diktyszitates bei IA durch den Hinweis auf die notorische Lückenhaftigkeit der Wiener Troika zu erklären gesucht. Gleichwohl muß es Kedren aus Malalas genommen haben. Mal. 107, 1 sqq. heißt es: xaìs $\delta$

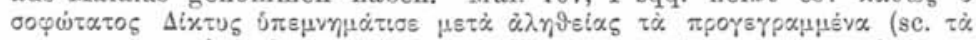

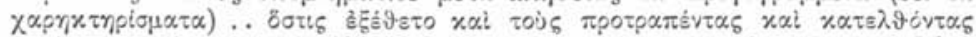

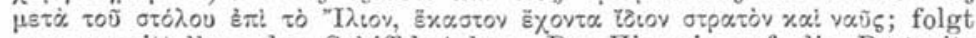
nun unmittelbar der Schiffskatalog. Der Hinweis auf die Portraits und die Erwähnung des Schiffskatalogs ist beiden gemeinsam. Bei Kedren muß die Anführung dieser zwei speciellen Punkte aufs höchste befremden. Denn er hat weder die Heldenportraits noch auch den catalogus. Wozu aber die Berufung auf einen Augenzeugen für Dinge, die in dereigenen Erzählung garnicht berührt werden? Ein Blick auf den Zusammenhang bei Malalas macht uns die Sache klar. Hier steht nämlich das Zitat gerade zwischen die Personalschilderungen und den Schiffskatalog eingekeilt. Bei ihm ist es also durchaus sinnhaft und verständlich: aus ihm hat es Kedren ohne Berücksichtigung seines von Malalas abweichenden (gekürzten) Kontextes unverändert herübergenommen. Bei IA kann das Zitat in der Form des Kedrenos nicht vorausgesetzt werden. Denn von den Heldenportraits hat er keine Spur und den Schiffskatalog an anderer Stelle als Malalas. Dazu gesellt sich ein weiteres Moment. Bei Kedren steht das Zitat unmittelbar hinter der Polydorepisode, die mit den Streifzügen der Griechen in die Nachbarschaft Troias eng zusammenhängt. Polydor wird ja auf einer solchen Expedition gefangen genommen. An diesen incursiones erscheint aber Idomeneus bei IA nicht beteiligt. Er hatte also jedenfalls nicht in gleichem Maaße Anlaß, sich hier auf

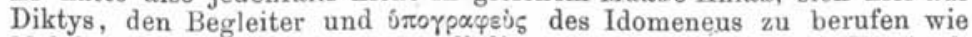
Malalas, der den Idomeneus an all diesen Zügen teilnehmen läßt. Auch 
etwa Homer selbst, ist nicht ausgeschlossen, wenn auch die homerische Patroklie wirklich aus IA herrühren sollte (Patzig, Byz. Ztschr. II 426). Diesem Ergebnis ist völlig entsprechend, was wir über die Herkunft der Kedren'schen oúvołıs im allgemeinen wissen. Gelzer hat dargethan, daßs für dieselbe einschlieflich der Troica der cod. Parisin. 1712 eine Hauptquelle sei $^{73}$ ). Der Parisinus selber ist aber eine ganz bunte Kompilation, wie Patzig gezeigt hat $\left.{ }^{73 n}\right)$.

Die Kedrenhypothese Noacks hat also bei genauerer Prüfung nicht Stich gehalten. Von einer engeren Verwandtschaft mit Septimius-Diktys ist überall nichts $\mathrm{zu}$ entdecken. Die

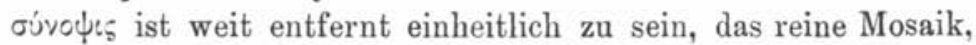
speciell die Troika setzen sich vorwiegend zusammen aus Ma-

erzählt IA die Polydorepiso de an einem ganz anderen Orte als Malalas (und Kedren). - Zu den sonstigen Beweisen, die Noack für seine Kedrenhypothese ins Feld geführt hat, habe ich nicht mehr viel zu bemerken. Die angebliche Uebereinstimmung des Kedren mit Johannes Nikiu, einem Ausschreiber des Malalas, beweist nichts. Denn der $\mathrm{Zu}$ satz zu $\chi \alpha \rho \tau \dot{\alpha} \gamma \alpha t v \alpha$ (Kedren 246, 15: spiel jener byzantinischen Marotte, fremden, wenn auch noch so bekannten Eigennamen und technischen Ausdrücken ihre unvermeidlichen

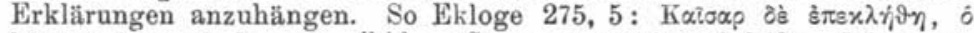

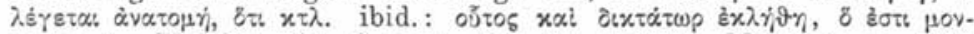
apxns (= Malalas 214, 2 sqq.; Joel 24, 4 sqq.). Manasses v. 1800 u. a. Wenn nun zwei Autoren in einer derartigen Worterklärung einmal zusammentreffen, was hat denn das Auffallendes? Wer immer sich vornähme, das X $\alpha$ p $\dot{\alpha} \gamma \alpha$ เv $\alpha$ zu erklären, müßte doch auch auf eine materielle gleiche Deutung kommen. Welche Fassung sie aber näherhin $\mathrm{im}$ griechischen Original des Joh. Nikiu gehabt habe, können wir nicht wissen. Daß dieser Zusatz im vollständigen Malalas gestanden habe (Patzig Byz. Ztschr. II 424), brauchen wir also nicht einmal anzunehmen. - Was jene Verwechslung der beiden Städtenamen 'A $\beta \beta \alpha$ vi $\alpha$

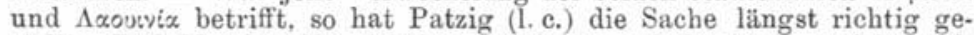
stellt. Daf Malalas die richtige Zuteilung gehabt hat, ergiebt sich aus der Parallelstelle Ekloge 222 , die Noack und Patzig übersehen haben. Hier ist $\Lambda$ oovevi $\alpha$ 'A $\lambda \beta \alpha v i \alpha$ die Stadt des Ascanius. Außerdem finden wir auch in den Excerpta lat. Barbari ed. C. Frick, Chronica minora p. 184 sqq., die sicherlich von Malalas abhängen (Frick l. c. CLXII), die richtige Appropriierung. An derartigen Verschreibungen von Eigennamen ist der Oxoniensis überreich, wie der kritische Apparat bei Dindorf erkennen läßt. Ich gebe einige Beispiele per saturam.

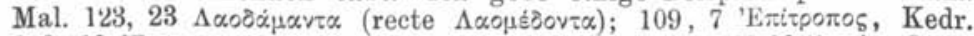

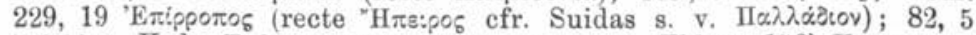

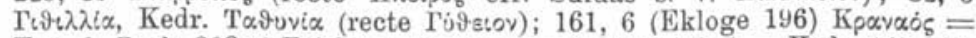
Exc. 1. Barb. 308, 6 Frick (recte K $\alpha$ pavo cfr. Mal. 72, $1=$ Kedr. 145, 13).

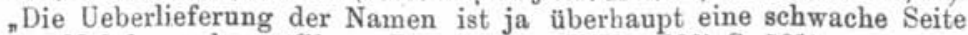
des Malalaswerkes“ (Gleye, Byz. Ztschr. VIII (1899) S. 501).

$\left.{ }^{73}\right)$ II $357 \mathrm{ff}$; vgl. nun auch Patzig, Byz. Ztschr. IX $204 \mathrm{ff}$.

${ }^{73 a}$ ) Byz. Ztschr. I 138; Progr. 1892 S. 18. 
lalas und IA. Ihre Bedeutung für die Ableitung des Diktysstoffes liegt darin, dafs diese beiden Byzantiner in vollständigerer Form (vgl. z. B. Kedren 233, 7 mit Mal. 121, 17 u. Ekloge 213, 7) zu Grunde liegen.

\section{Der Diktysstoff bei den übrigen Byzantinern.}

Der Vollständigkeit wegen gebe ich nachstehend eine übersichtliche Zusammenstellung derjenigen byzantinischen Autoren, bei denen irgendwelche Spuren des griechischen Diktys $\mathrm{zu}$ finden sind.

1) Konstantin Manasses. In dessen in politischen Versen geschriebener Chronik stehen die Troica v. 1107 bis 1471. Nach Hirsch, Byz. Studien ${ }^{74}$ ) soll er ein Auszug aus Glykas-Skylitzes-Zonaras sein. Patzig ${ }^{74 a}$ ) hat aber überzeugend nachgewiesen, daßs die Troica in specie aus IA geschöpft sind. Dieses Abhängigkeitsverhältnis wird durch die neuen Troica des IA vollauf bestätigt ${ }^{75}$ ). $\mathrm{Ob}$ aber nicht daneben noch andere Quellen (Greif S. 265 f. vgl. v. 1110) etwa Malalas (Noack a. a. O. S. 496) benützt sind, läbt sich mit Sicherheit nicht entscheiden. Noack hat ihn mit Unrecht fast gänzlich ignoriert. Durch die Auffindung der Wiener Troika ist indes seine Bedeutung als Ergänzung des IA erheblich gesunken.

2) Die Hypothesis der Odys e e aus dem codex Palatinus (XIV saec.) hrsg. v. Dindorf, Odysseescholien I 1855 p. 3 f. Sie wurde erstmals herangezogen von Noack (S. 405), der sie auf seine Sisyphoschronik zurückführte. Allein diese haben wir ablehnen müssen. Patzig (Byz. Ztschr. II 424) meint, die Hypothesis stamme in ihrer ganzen Ausdehnung wörtlich aus der Chronik des IA und sei das umfangreichste Stück, das uns aus diesem erhalten ist. Diese Thesis läft sich nicht mehr halten, seitdem wir den Wiener Johannestext kennen. Denn wenn weder dieser noch die früher bekannten IA excerpte (mit Ausnahme der salmasischen, vgl. die folgende

$74)$ S. 404.

74a) Byz. Ztschr. I 139 Progr. 1892 S. 5. 12. 17. Byz. Ztschr. II 414.

$\left.{ }^{75}\right)$ Traum der Hekuba (v. 1120) mit IA übereinstimmend; ebenso die Datierung der Troica unter David v. 1107.

Philologus LX (N. F. XIV), s. 
No. 3), die da einsetzen, wo jener abbricht, keine Spur von Sisyphos verraten, so ist so gut als gewiß, dafs Sisyphos von IA nicht benützt und darum auch nicht genannt wurde. Also muj doch wohl noch eine andere Quelle postuliert werden. Sie brauchen wir nicht weit zu suchen: es ist Malalas gewesen, den der Verfasser der Hypothesis neben IA ausgeschrieben hat. Der Beweis liegt im folgenden. Noack hat a. a. O. ausgeführt, wie stark die Hypothesis sich mit Malalas berührt. In mehreren Teilen stimmt aber auch Kedren wörtlich überein. Gleichwohl können diese Partien nicht aus Kedren noch aus Malalas allein hergeleitet werden. Denn von Kedren werden sie getrennt durch das Auftauchen des Sisyphos und Malalas gegenüber bieten sie einen Ueberschuss $\left.{ }^{76}\right)$. Dieser besteht vor

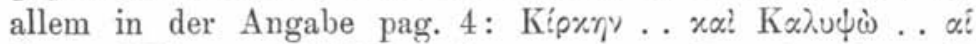

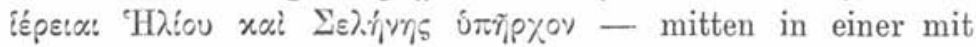
Malalas identischen Umgebung. Dieser letztere Umstand weist uns auf einen Autor, der selbständig aus einer mit Malalas gemeinsamen Quelle schöpfte: das ist Johannes von Antiochien. Nun enthält aber auch das salmasische Excerpt jene Angabe über Kirke und Kalypso: auch in ihm ist Sisyphos zitiert und besteht überhaupt eine innige Beziehung zwischen ihm und der Hypothesis, wie die folgende Gegenüberstellung zeigt.

Dind. 4, 12-5, 2 (Hyp.)

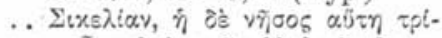

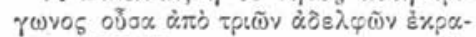

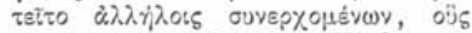

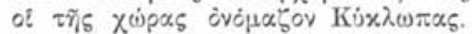

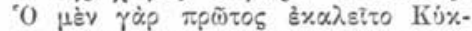

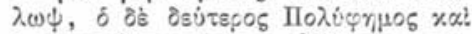

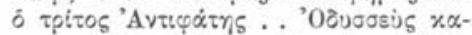

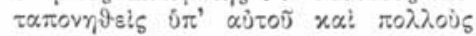

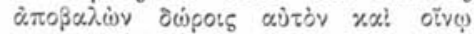

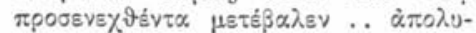

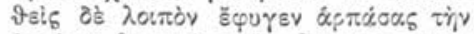

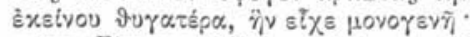

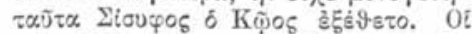

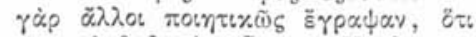

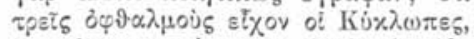

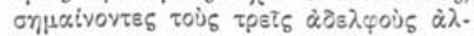

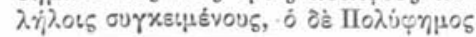

Cramer 1. c. $391,5-14$ (Salm.)

'H $\Sigma: x \varepsilon \lambda i \alpha$

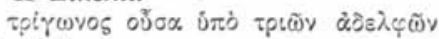

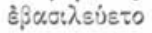

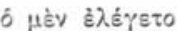
$\bar{\omega} \%$

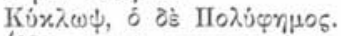

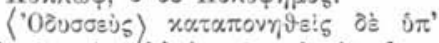

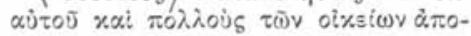

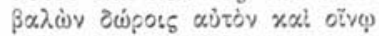

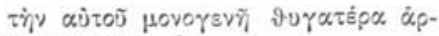

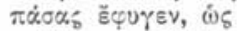

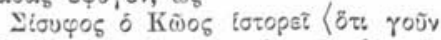

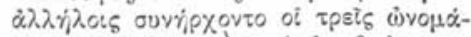

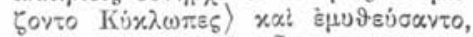

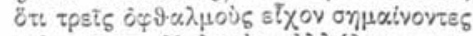

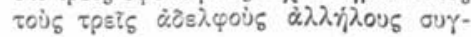

${ }^{76}$ ) Auch sagt Noack a. a. O., die Hypothesis könne diese Stücke nicht unabhängig von Kedrenos aus Malalas fast wörtlich so hergestellt haben, wie sie Kedren aus anderer Quelle zusammenschrieb. Dieses Argument fällt für uns ohnehin weg, da ja Kedren von Malalas selbst abhängig ist. 


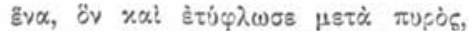

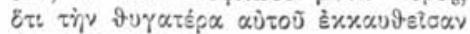

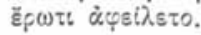

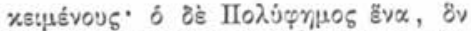

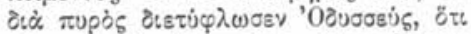

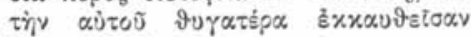

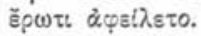

Es ist nicht wahrscheinlich, daß eine solche Uebereinstimmung möglich war, wenn diesé beiden Berichte unabhängig von einander aus Malalas und IA zugleich oder aus Malalas allein geschöpft wurden. Wir müssen vielmehr eine Quelle ansetzen, in der dieser Ausschnitt aus dem byzantinischen Diktysbericht die in der Hyp. und in den Exc. Salm. sich findende Form erhalten hat. Es ist wohl keine andere als jene, auf welche bereits Sotiriades ${ }^{77}$ )

3) die salmasischen Excerpte $\mathrm{e}^{78}$ ) zurückgeführt hat: die Schrift eines Anonymus, vermutlich des 9. Jahrhunderts $\left.{ }^{79}\right)$. Dieser selbst soll nach Sotiriades aus Malalas herstammen, mit dem sich die Salmasiana in der That fast durchgehends berühren ${ }^{80}$ ). Nur findet sich bei diesen eine Notiz betreffend die Benennung des ägäischen Meeres nach Aigeus, die aukier Kedren 215, 13 nirgends zu belegen ist; ferner die Angabe, daf Kirke und Kalypso Priesterinnen nicht blok des Helios (= Malalas 117, 20; Ekloge 210, 20), sondern auch der Selene ( $=$ Hypothesis pag. 4) gewesen seien. Dieses plus vermissen wir bei Malalas. Also muf sich der Anonymus noch an eine andere Vorlage gehalten haben, die aber selbst wieder mit Malalas verschwistert war: Johannes Antiochenus. Beweis ist die eben bemerkte Uebereinstimmung mit Kedren ${ }^{81}$ ), der ja den IA benützte, besonders aber die folgende Vergleichung:

Salm. 390, 19 sqq. (Cramer)

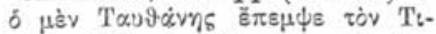

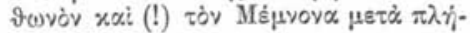
งัoงร 'Iทชิ์ข.
IA 7,15 sqq. (Heinr.)

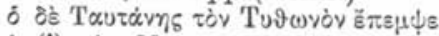

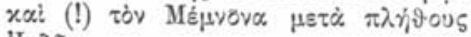
'Iขอิธัข.

77) A. a. O. S. 24 f.; 37.

${ }^{78)}$ Text bei Müller FHG IV fr. 24. Cramer 1. c. II $390 \mathrm{~s}$.

$\left.{ }^{79}\right)$ Auch C. de Boor Byz. Ztschr. II 1893 S. 208 leitet die Salmasiana aus einem "namenlosen Kompendium" her; gegen ihn freilich Patzig Byz. Ztschr. II 591. - Auf dieser Linie bewegt sich auch A. Ludwich, Zwei byzantinische Odysseuslegenden, Vorlesungsverzeichnis der Univ. Königsberg 1898 S. 2 f., wenn er einen Unbekannten die Hypothesis aus Malalas schöpfen läßt.

$\left.{ }^{80}\right)$ Vgl. den Nachweis bei Noack S. 433.

${ }^{82}$ ) Vgl. IA fr. 24 no 8 (Salm.) mit Kedren 230, 1 (Patzig, Byz. Ztschr. IV S. 27). 
Also beide geben die befremdliche Notiz, dafs Tithonos sowohl als auch Memnon den Troianern zu Hilfe gezogen seien. Septimius IV 4 weißs nur von einem Zuge des Memnon allein und schon Lehrs ${ }^{82}$ ) hat gegen Dunger ${ }^{83}$ ) treffend ausgeführt, daßs im Diktysbericht überhaupt von einer Betheiligung des Tithonos nie etwas gestanden hat. Interessant ist

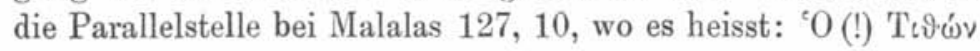

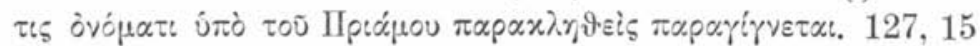
und dann im folgenden immerfort ist sein Name wieder plötzlich Memnon. Malalas meint ohne Zweifel den Memnon allein; aber Thatsache bleibt, dak die Stelle in Unordnung ist Das legt die Annahme nahe, daf in der Vorlage des Malalas eine mißverständliche Wendung ${ }^{84}$ ) sich fand, der die beiden Epitomatoren Malalas und IA - aber jeder in verschiedener Weise - zum Opfer fielen. Wenn nun das salmasische Excerpt diese mifverstandene Stelle genau in der Fassung des IA wiedergiebt, so ist $\mathrm{m}$. E. bewiesen, daßs der Anonymus, auf den es zurückgeht, neben Malalas auch IA benützte. Es ergäbe sich demnach folgendes Schema:

$\overbrace{\text { Hypothesis }}^{\frac{\text { Malalas }}{\text { Anonymus }}} \frac{\text { IA }}{\text { Salmas }^{84 a} \text { ). }}$

4) Die Homerscholien. Hier kann ich mich kurz fassen. Noack, der sie als einen nicht zu verachtenden Beweis für die Existenz eines griechischen Diktys herangezogen hat, hat sie so erschöpfend behandelt als nach dem Stand der Sache überhaupt möglich ist. Bei Scholion T 206 zweifelt Noack (S. 493) bezüglich der Provenienz aus Diktys. Um so sicherer

$\left.{ }^{82}\right)$ Wissenschaftl. Monatsbl. VI 1878 S. 132 f.

$\left.{ }^{83}\right)$ Diktys-Septimius S. 21.

$\left.{ }^{84}\right)$ Lehrs a. a. O. S. 133.

84a) Wenn Greif, Neue Untersuchungen S. 24 die Salmasiana unter Berufung auf de Boor, Hermes XXXIV (1899) S. 298 ff. der Sphäre des Antiocheners gänzlich entrücken will, so verweise ich ihn auf die gehaltvollen Darlegungen Patzigs Byz. Ztschr. IX S. $357 \mathrm{ff}$. Ich konnte von ihnen vor Beginn der Drucklegung nur noch flüchtig Kenntnis nehmen, gestehe aber, daß sie mich in meiner hier skizzierten Ansicht wieder schwankend gemacht haben; vgl. auch die vorige Nummer und dazu jetzt Patzigs Bemerkungen Byz. Ztschr. IX 523 ff., die ich nur noch zitieren kann. 
aber weisen die Doppelnamen Chryseis-Astynome und BriseisHippodameia auf Diktys hin ${ }^{85}$ ). Für die Erzählung des Iphigenieopfers wird Diktys ausdrïcklich zitiert (Scholion A zu A 107). Für die Herkunft aus Diktys ist, wie ich glaube, der Umstand ausschlaggebend, daß3 das von Agamemnon per nefas erlegte Tier nicht $\tilde{\varepsilon} \lambda \alpha \varphi^{\circ} \varsigma$, sondern wie im Diktysbericht aik (Kedr. 219, 12; Sept. I 19) genannt ist. Dafs der Scholiast auf einer byzantinischen Fassung des Diktysberichtes fusse, ist durch keine auch noch so schwache Spur indiziert. Und so bin ich mit Noack wirklich der Ansicht, dafis Schol. A. 108 und 392 auf das griechische Diktysbuch direkt zurückzuführen sind.

5) Johannes Nikiu ${ }^{86}$ ). Noack (S. 186) glaubte die Troiaerzählung dieses Chronisten auf seine Chronik A I direkt zurückführen zu dürfen. Den Troica selbst konnte er einen Beweis freilich nicht entnehmen. Denn diese sind im Zustand hoffnungsloser Trümmerhaftigkeit überliefert, und soweit sie einen Schluf ermöglichen, bewegt er sich in ganz anderer Richtung. Die trojanischen Geschichten verteilen sich auf die Kap. XLV, XLVI, XLVII. Ich mufs zur Begründung des Gesagten den Text resp. die Glossen Zotenbergs hier folgen lassen. Notices et Extraits t. 24. 1. p. p. 386, Chap. XLV Zotenberg not. 5: Il est impossible de trouver dans ce texte corrompu une narration raisonnable. Dans les noms propres également fort altérés, on croit reconnaître les noms d'llion, de Priame, de la Phrygie, de Sparte. Chap. XLV : Il fut un homme, nommé Palamédès, plein de sagesse et de science, qui le premier enseigna l'art de musique, la viole, la lyre, la eithare et tous les instruments de musique ${ }^{87}$ ). Chap. XLVII Zotenberg not. 7: Ce chapitre, plein de contresens, est un fragment de la guerre de Troie. Dans la première phrase on

${ }^{85}$ ) Cf. Malalas 100 D. Derartige Dyonymien scheinen zum Inventar dieser Schwindellitteraten gehört zu haben. Auch bei Ptolemaeus-Chennus und Pseudoplutarch ziemlich übereinstimmend vgl. Hercher, Fleckeisens Jahrbücher a. a. O. S. 281. Auch den papyri ist diese Erscheinung nicht fremd; vgl. noch R. Herzog im Philolog. 56 (1897) S. 56 u. 35 A. 3.

${ }^{86)}$ S. Krumbacher, Byz. Littg. ${ }^{2}$ S. 404.

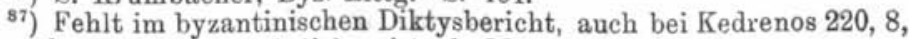
der noch ziemlich ausführlicher ist als Malalas. 
reconnait facilement la traduction altérée et tronquée d'un passage grec qu'on lit dans la chronique de Malalas col. 200

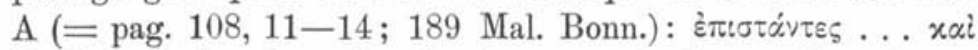

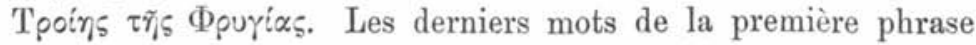
et la seconde phrase sont un fragment de l'histoire du Palladium (Mal. col. $200 \mathrm{~B}=109,4-8$ Mal. Bonn: $x \alpha \imath$ ن́ $\pi \dot{\rho} \rho$ $\varepsilon \dot{y} \% \alpha \rho t \sigma \tau i \alpha \varsigma . . \mu \varepsilon \tau \varepsilon x \dot{\lambda} \lambda \varepsilon \sigma \varepsilon v)$. Enfin la dernière phrase et les derniers mots de l'avantderrière proviennent du récit des aventures d' Ulysse en Sicile. Je pense que (äthiopisches Wort)

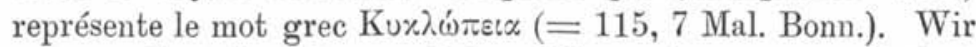
sehen, selbst diese kümmerlichen Ueberreste zeugen noch laut genug für die Abhängigkeit von Malalas, die auch bis auf Noack unbestritten war ${ }^{88}$ ). Noack's Einwände haben wir bereits oben im Zusammenhang mit seiner Kedrenhypothese erledigt. Weit entfernt eine noch reinere Form der Diktysüberlieferung als Kedrenos darzustellen, ist der Troiabericht des Joh. Nikiu nichts als ein Abklatsch des Malalas und ohne selbständige Bedeutung.

6) Suidas. Es unterliegt keinem Zweifel, dafi in meh-

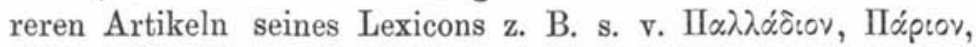

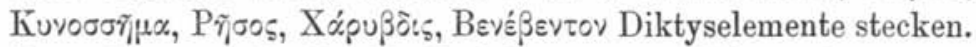
Von einer unmittelbaren Entlehnung aus Diktys kann von vornherein nicht die Rede sein. Patzig wies das sämtliche Diktysgut des Suidas dem IA zu (vergl. aber Krumbacher B. Littg. ${ }^{2}$ S. 565 und 567), während Noack (S. 433, 486) unsern Autor mit Kedren in ein e Reihe $\mathrm{zu}$ bringen und damit eine weitere Stütze für seine ganze Hypothese zu erlangen suchte. Der Versuch scheint mir mißlungen zu sein und ich glaube, dafs die oben genannten Artikel überhaupt nicht auf ein und dieselbe Quelle zurückzuführen sind. Von den beiden nämlich, die wir allein mit IA ed. Heinr. vergleichen können, Шáptov und $\Pi \alpha \lambda \lambda \dot{\delta} \delta: \circ v$, ist der erstere aufs nächste mit Mal. 92, 13 verwandt, der letztere aber so völlig mit IA 4,9 identisch, dafs wir die beiden Stellen ohne weiteres vertauschen können. Bei diesem Stand der Sache habe ich mich nicht zu entscheiden gewagt.

7. Johannes Tzetzes. Er hat den Diktys wieder-

${ }^{88)}$ Vgl. Nöldeke, Götting. gelehrt. Anz. 1881 S. 587; 1893 S. 1364. Patzig Progr. 1892 S, 23 ff. Krumbacher B. Littg. a. a. 0. 
holt zitiert (Epigr. 30 der V Chil., v. 61 sq. in den Scholien zu Lykophron, die andern Zitate mit letzterem identisch). Direkte Benützung des Diktysbuches hat schon Körting ${ }^{89}$ ) abgelehnt. Nach Greif ${ }^{90}$ ) schöpfte er aus Malalas, nach Patzig ${ }^{91}$ ) aus den Konstantinischen IAexcerpten, eine Ansicht gegen die das Sisyphoszitat Chil. V 829 sqq. geltend zu machen ist. Noack hat im Hermes 27 (1892) S. 463 den Nachweis geliefert, daf die Iliupersis in den Posthomerika (v. 634-650 674-749) aus Tryphiodor fast abgeschrieben ist, während der vorhergehende Teil der Posthomerika in Quintus Smyrnaeus + Malalas fast ohne Rest aufgeht. Auf den letzteren werden wir getrost das gesamte Diktysgut des T. zurückführen dürfen.

8. Der codex Parisinus 1630 . Das Excerpt aus ihm (Müller FHG IV 540 fr. 2 u. not. 2 ; Cramer (l. c. p. 379) bleibt für die Diktysforschung aufer Betracht ${ }^{92}$ ). Wenn Patzig ${ }^{93}$ ) mit Hilfe dieses Fragments die Hypothesis des Palat. restlos aus IA ableiten wollte, so ist das bereits durch no 2 und 3 erledigt.

9. Die Weltchronik des Simeon Magister enthält keine Troika. Uebrigens ist der echte Symeon M. noch gar nicht ediert. Nach Krumbacher Byz. Littg. ${ }^{2}$ S. 358 soll er berufen sein, „für die Aufklärung der labyrinthischen Quellenverhältnisse der späteren Chronisten eine große Rolle zu spielen". Hoffen wir, daki auch für die Entwirrung der byzantinischen Diktystradition etwas abfällt!

10. Die Weltchronik des Georgios Mona$\mathrm{ch}$ os (Hamartolos). Seine abgerissenen Andeutungen über die Troica kommen kaum in Betracht und könnte ihre Herkunft nur im Zusammenhang mit der Erforschung seiner übrigen Quellen eruiert werden. Das chaotische Durcheinander gerade der ersten 3 Bücher ist noch nicht einmal notdürftig gelichtet ${ }^{94}$ ).

11. Die Synopsis Sathae. Es ist ediert in $\mathrm{Me}-$

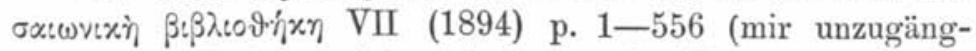
lich). Die Troica finden sich S. 5-19. Ueber die für uns

$\left.{ }^{89}\right)$ Dikt.-Sept. S. 42 ff.

${ }^{90}$ ) S. 219 u. 259.

${ }^{91}$ Byz. Ztschr.

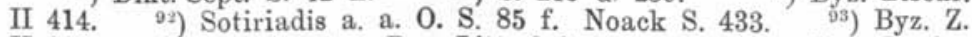

II 216. ${ }^{94}$ ) Krumbacher, Byz. Littg. ${ }^{2}$ S. 354 vgl. Gleye Byz. Ztschr. IV 157. Die Untersuchungen Sestakov's (Byz. Ztschr. II 166) sind mir unzugänglich. 
ziemlich irrelevanten Abstammungsverhältnisse s. Patzig, Byz. Ztschr. V 1896 S. 182 ; vgl. Krumbacher, Byz. Littg. ${ }^{2}$ ) S. 388.

12. Die Chronik des Synkellos bietet S. 322 , 1-22 B. eine ganz dürre Erzählung des troianischen Feldzugs. Ohne Belang.

13. Die übrige Chronographenlitteratur (von den im Laufe der Untersuchung bereits genannten Werken wie Kedrenos und Wiener Johannes abgesehen), in der sich entweder gar keine oder höchst dürftige Bemerkungen über den troischen Krieg finden, kommt für die byzantinische Diktysüberlieferung weiter nicht in Betracht ${ }^{95}$ ).

Ich glaubte, diese skizzenhafte Uebersicht im Interesse einer genügenden Orientierung über meine Ergebnisse anfügen zu sollen. Den ganzen Abschnitt aber möchte ich nicht schliefen, ohne meinen Standpunkt durch ein eigenes Stemma veranschaulicht $\mathrm{zu}$ haben:

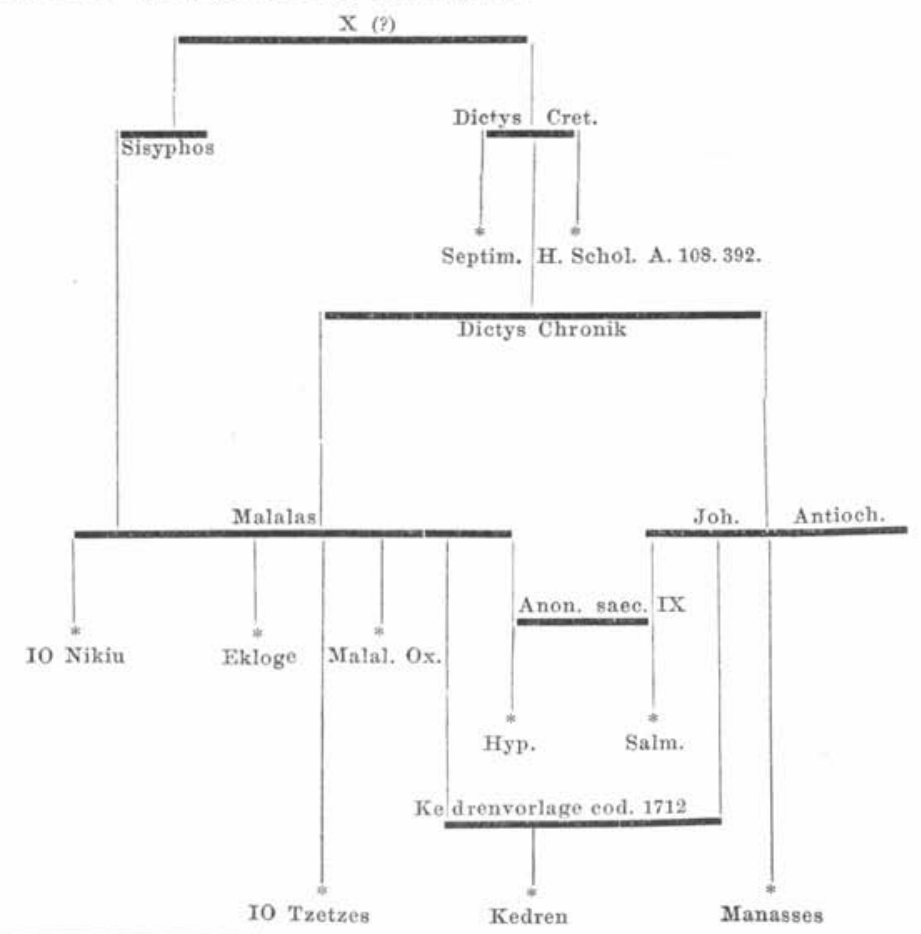

${ }^{\text {o5) }}$ Z. B. Osterchronik, Joel, Glykas u. a. 
VI. Ueber einzelneStückeder (byzantinischen) Diktysüberlieferung.

1. Der Prolog und die Widmungsepistel. Der Prolog enthält die Fabeleien über das Schicksal und die Wiederauffindung des griechischen Diktysbuches ${ }^{96}$ ). Ganz übereinstimmend, nur kürzer sind sie von Malalas 107, 1 sqq. wiedergegeben. Wir werden ihn also dem griechischen Diktysoriginal $\mathrm{zu}$ vindizieren haben ${ }^{97}$ ). - Septimius hat seine Uebersetzung einem gewissen Qu. Aradius gewidmet. Die Dedikationsepistel ist eine Kopie des Prologs, erweitert durch Angaben über die Methode, nach der die Uebersetzung gearbeitet ist. Die Echtheit derselben wurde wie die des Prologs viel umstritten ${ }^{98}$ ). Nachdem die Existenz eines griechischen Diktys erwiesen ist, hat man keinen Grund mehr, den Widmungsbrief dem Septimius abzusprechen ${ }^{99}$ ).

2. Der Traum der Hekuba. Oneirokrisis. Der Traum fehlt bei Malalas und Kedren. Septimius berichtet nicht nur den Traum, sondern auch dessen Auslegung durch die haruspices (p. 68, 19). Es fragt sich nun, ob jedes oder wenigstens das eine oder das andere von beiden Momenten bei den Byzantinern und also auch im Urdiktys gestanden habe. Die Frage ist in ihrem vollen Umfang zu bejahen.

$\left.{ }^{90}\right)$ Ein Analogon bietet der Widmungsbrief des Romanschreibers Antonius Diogenes, vgl. Rohde, d. gr. Roman S. 252.

${ }^{97}$ ) Hertzberg, die Quellen der Troilussage (Jahrbuch der deutschen Shakespeare-Gesellschaft VI (1871) S. 174. 179 läßt seltsamerweise den Prolog $\mathrm{n}$ a $\mathrm{ch}$ der Epistel entstanden sein.

99) Pro: besonders Dederich vor seiner Ausg. XII sq.; contra: Perizonius $\S 30,31$ sqq. ; Hildebrand Jahns Jahrbb. XXIII.

${ }^{99}$ ) Greif, Neue Untersuchungen S. 6 f. hat die zwischen Prolog und Epistel bestehenden Differenzen mit allem Nachdruck betont. Sie seien von dem Fälscher Septimius mit besonderer Schlauheit ausgeheckt worden, um die Ueberzeugung zu erwecken, daß der Verfasser des Prologs mit dem der Epistel nicht identisch sei. Ich muß gestehen, daß ich in dem Verhältnis von Prolog und Epistel überall nichts von Tendenz und Raffinement zu entdecken vermag. Die Epistel macht auf jeden unbefangenen Leser den Eindruck einer flüchtigen und gedächtnismäßigen Recapitulation der im Prolog gemachten Angaben. Sie ist natürlich nach Vollendung der ganzen Uebersetzungsarbeit geschrieben. Es ist gar nicht zu verkennen, wie die Epistel gleichgültig und in fast atemlosem Lauf über das bereits im Prolog Mitgeteilte hinweg den Schlußbemerkungen zueilt, auf die es in der Epistel ganz ausschließlich ankam. 
Denn nicht blok das Fragm. 23 (Müller FHG l. c. p. 550) ${ }^{100}$ ) und Manasses v. 1121-1124, sondern auch der neue IA text des cod. Vind. $(4,2)$ bieten den Traum $\left.{ }^{101}\right)$. Auch die Traumdeutung hat der byzantinische Diktysbericht nachweisbar enthalten, eine Thatsache, die Noack S. 468 weit zuversichtlicher hätte konstatieren können, wenn er nicht wiederum den (von IA abhängigen) Manasses ignoriert hätte $\left.{ }^{102}\right)$. V. 1226 fährt

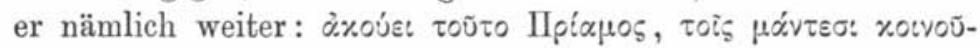

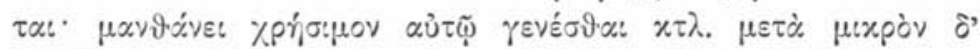

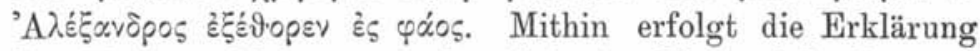
des Traumgesichtes vor der Geburt. Man wird also mit gutem Grund die an sich zweifelhaften Worte des IAfragm. ${ }^{103}$ ):

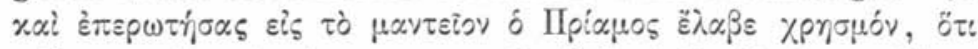
$\chi \tau \lambda$. von einer Auslegung vor der Geburt des Paris verstehen. Ein Irrtum von Noack ist es, wenn er a. a. O. sagt: „Als Neuerung sehen wir im Diktysbericht nur die Verdopplung der Deutung des Traumes vor und nach der Geburt des Paris an. " Nirgends in der byzantinischen 'Tradition ist eine doppelte Auslegung bei einem und demselben Schriftsteller anzutreffen. Vielmehr ist das Verhältnis dieses:

V or der Geburt.

IA fragm. 23.

Manasses v. 1120 sqq.

Tzetzes Antehom. 43 sq.

Euripides Alexandros (s. G.

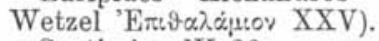

Septimius III 26.
$\mathrm{N}$ a c h der Geburt.

Malalas 92,1 sqq.

Kedren 216, 13 sqq.

Es handelt sich also je nur um einen einzigen Vorgang, der von Malalas und bezeichnenderweise auch von Kedren zeitlich verrückt wurde. Dafs Septimius und die mit ihm harmonierende Majorität der Griechen die ursprüngliche Version bieten, ist kaum zu bezweifeln. Es ist an sich schon natürlicher, daßs für ein so dunkles, v or der Geburt erhaltenes Traumgesicht noch vor derselben auch der Bescheid des Orakels eingeholt werde. Noch ein Wort über den Standort des Traumes im griechischen Diktys! Bei Septimius III 26

$\left.{ }^{100}\right)$ Vgl. Patzig, Byz. Ztschr. II S. 423.

$\left.{ }^{101}\right)$ Vgl. Gleye, Byz. Ztschr. V 453; Praechter ebda. IV 542.

$\left.{ }^{102}\right)$ Vgl. auch die Troijanska prié, Archiv f. slav. Philol. XX (1898) S. 611.

${ }^{103}$ ) Der Wiener Johannes hat von der Oneirokrisis nichts. 
ist er in die Hektorlysis verflochten, die Byzantiner dagegen erzählen ihn in der Jugendgeschichte des Paris. Dahin gehört er auch naturgemäf. Septimius hat sich in diesem Passus verschiedene Aenderungen erlaubt (Noack S. 468) und die getreuere Wiedergabe ist hier sicherlich auf Seiten der Byzantiner.

3. Paris in Sparta. Septimius I 3 kommt Paris erst nach der Abfahrt des Menelaus nach Sparta. Die gesamte übrige Tradition läßt ihn noch während der Anwesenheit des M. eintreffen und trennt sich erst wieder bezüglich des Bekanntwerdens mit der Helena. In den rumänischen Troika (Byz. Ztschr. III 532 no 5) verliebt sich Helena in Paris noch in Anwesenheit ihres Gatten (Kypria und Ovid, Heroides XVII 83), dagegen bei Malalas 95, 4, IA 5, 15, Kedren 217, 18 wieder mehr im Einklang mit Septimius erst nach der Abreise des Königs. Mafgebend ist für diesen ganzen Passus zweifellos die byzantinische Erzählung. - Nach Kedr. 217, 18 ging Helena gerade im „Garten ihres Schlosses“ spazieren, als sie von Paris erblickt wurde - „damit wir ja an die Sultanin denken" (Lehrs, Populäre Aufsätze S. 19);

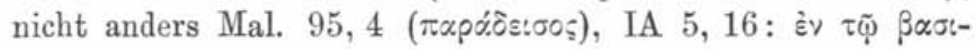

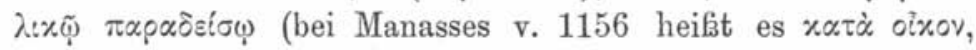
vgl. etwa Rohde S. 382). Ob wir in dieser Wendung wirklich mit Lehrs eine byzantinische Ausschmückung zu erkennen haben, möchte ich stark bezweifeln. Sie würde nicht übel passen zu den romanhaften Zügen, die dem griechischen Diktys allem nach eigen gewesen. Ich habe verhältnismäßig nur wenige Stellen aus dem Roman mit Diktys verglichen, aber dennoch frappante Anklänge gefunden. Schon Rohde (S. 391. A 3) hat bemerkt, wie Xenophon Ephesinus trotz der sonst so rasch dahineilenden Erzählung immer noch Platz findet, „um die nach unserer Vorstellungsweise so unwichtige feierliche Beisetzung Verstorbener verhältnismäßig breit zu erzählen ". So III 2, 13. V 10,3. V 15,3; genau so in Philostrat's "Geschichte des Apollonius von Tyrus" p. 29, 8; 30, 14; 35, $13 ; 38,14$. Mit welch groker Sorgfalt Diktys die funera verzeichnet und zwar zuweilen sehr ausführlich, mag aus der Zusammenstellung folgender Stellen ersehen werden: Sept II 
2. 12. 15. 27 (vgl. Kedren 220, 7). 32. 39. 41. III 9. 12. 14. 17 . 27. IV 1. 8. 9. 13. 15. 18. 21. V 13. 15. 16. VI 13. - Mit dem allgemeinen Romanapparat berührt sich Diktys besonders durch die Einführung zahlloser Reden: Sept. I 6 (p. 6, 13 u. 19 M). 9. 11. 15. 20. 21. II 5. 21. 23. 25. 26. (p. 34 u. 35). 28. 51. III 3. 21. 22. 24. 25. 26. 27. IV 15. 18. 22. V 2. Dak3 erst der Uebersetzer von sich aus diese Reden zusammengestoppelt oder auch nur wesentlich vermehrt habe (vgl. Brünnert, Sallust und Diktys, Gymnasialpr. Erfurt 1883 S. 18; Noack S. 466), halte ich nicht für wahrscheinlich. Denn auch die Byzantiner haben z. T. diese Reden mit Sept. gemein und haben noch Spuren von solchen, die Septimius, sagen wir, verschmäht hat (Kedren 229 sqq., vgl. Körting, D. u. D. S. 26). - Ein Lieblingsmotiv der Romanschreiber ist der Schiffbruch gewesen (vgl. z. B. Rohde 412 u. sonst, Norden, Antike Kunstprosa S. 409 ; vgl. Longin, Hirteng. I 31). Mal. 121, 14 wird der Schiffbruch des Odysseus in merkwürdigem Anklang an Xenoph. Ephes. 356, $26 \mathrm{X}$ erzählt:

\section{Mal.}

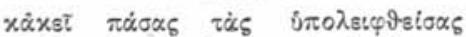

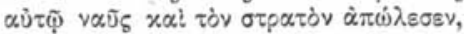

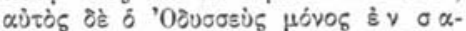

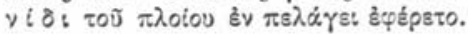

Xen. Ephes.

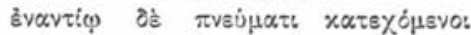

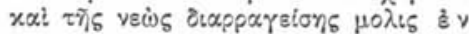

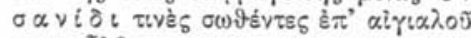

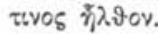

Sich auf den Zufall zu berufen ist dadurch erschwert, daß3 Malalas an dieser (homerischen) Stelle gerade in dem Punkt mit Xenoph. zusammentrifft, in welchem er von Homer (Od. XIII, 424 f.) abweicht. - Die Reise des Paris und der Helena mit ihren verschiedenen Abenteuern (Mal. 95, $12 \mathrm{ff}$., Sept. I 5) gemahnt mitunter an gewisse Scenen des Romans, vgl. Xen. Eph. 340, 13-17; 341, 6-11; 359, 20-361, 4; $369,17-22 ; 372,10-15 ; 373,14-374,10 ; 376,20$ f.; 379 $25-380,3 ; 382,13-15$; 383, 3-10. 23-25; 384, 17; 387, 20 f. ; 389, 7 f.; 392, 22-393, 7; 395, 6 f. Die Nennung Aegyptens in diesem Zusammenhang (vgl. allerdings Fuchs, de varietate p. 66) verstärkt die Aehnlichkeit (Xen. Eph. 339, 5; 371, 14). - Auch die Affaire beim Sidonierkönig (Mal. 95, 13. Sept. I 5) ist ganz im Geiste des Romans erzählt: Paris raubt und mordet im eigenen Hause des Königs - Volksauflauf - Kampf mit den Sidoniern - Flucht des Paares. Als 
dieses endlich in Troia eintrifft, fragen die Eltern des Paris

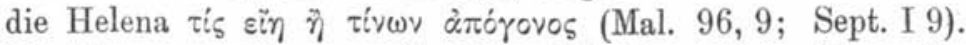
Der Roman hat in ganz ähnlicher Situation diesen Zug. So wird bei Heliodor die Chariklea um ihre Abkunft gefragt, vgl. Xenoph. Eph. p. 370. 376, 24. 384, 12 f. - Die unverkennbare Vorliebe des Diktys für Träume, Orakel, prodigia und portenta (Sept. I 21; V 7 [= Mal. 112, $1 \mathrm{ff}$.]; V 8; Mal. 112, 4; V 1, 14; IA 4, 2 Heinr.; Sept. III 26) berührt sich mit dem Roman (vgl. Rohde 49. 239, 1. 369. 371 u. a.). Die Beschreibung von Meerstürmen und Ungewittern ist eines jener Kunstmittelchen, deren sich die Romanschreiber bis zum Ueberdrufs bedienten (vgl. Norden, Antike Kunstprosa S. 286. 409 A.). Dak Diktys sich ebenfalls auf solche Sturmmelodien versteht, beweist I, 21: . cum ecce dies foedari et coelum nubilo tegi coepit; dein repente tonitrua, corusca fulmina et praeterea terrae marisque ingens motus atque ad postremum confusione aeris ereptum lumen. Neque multo post imbrium atque grandinis vis magna praecipitata. Inter quae tam taetra nulla requie tempestatis ect. (cfr. VI, 1); vgl. die bezeichnende Stelle bei Malalas 116, 6 ff., wo bei Erzählung von Odyss. IX $481+\mathrm{X} 87 \mathrm{ff}$. geflissentlich Töne aufgesetzt sind, die ganz an den "Morithaten"stil des Romans erinnern. Das sind einzelne ohne Wahl herausgegriffene Anklänge. $\mathrm{Ob}$ man ihnen Gewicht beilegen will oder nicht, auf alle Fälle weht bei Diktys die Luft des Sophistenromans. Wenn sie bei Septimius vielleicht weniger zu verspüren, so ist zu beachten, daf dieser erheblich gekürzt und geändert hat und daß3 der specifisch griechische Geist, der in der romanhaften Färbung zum Ausdruck kam, gerade am leichtesten unter der Hand des Römers sich verflüchtigen konnte. Alles in allem erwogen, hat Noack (Iliupersis 1892 S. 9) recht gethan, den griechischen Diktys in die Sphäre des Romanschreibers Philostratos zu rücken (vgl. dagegen Haeberlin Wochenschrift f. kl. Phil. 1891 S. 950). Das litterarische Milieu der troischen Schwindelbücher ist m. E. der griechische Roman. Eine eindringende Untersuchung dürfte hier noch manche Beziehungen bloflegen.

4. Die $\dot{\alpha} \pi \alpha i \tau \eta \sigma: \varsigma$ der Helena. Bei Mal. 97, 3 wird zuerst eine Gesandtschaft nach Troia geschickt, um die 
Helena zurückzuverlangen, an der sich aber Menelaus selbst nicht beteiligt. So auch IA 5, $36 \mathrm{H} \mathrm{u}$. Fragm. 23. Als diese

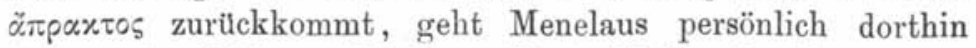

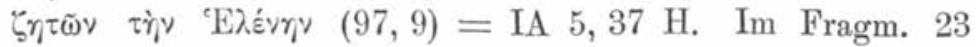
geht nach der ersten erfolglosen Deputation eine zweite ab

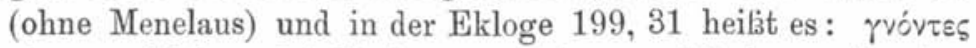

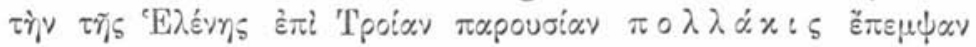

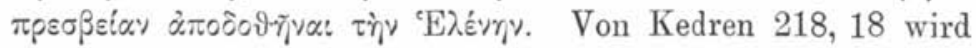
uns eine einzige $\pi \rho \varepsilon \sigma \beta \varepsilon i \alpha$ erwähnt, an der sich aber der Spartanerkönig in eigener Person beteiligt; ebenso Septimius I 4 p. 5, 16. Trotz aller Schwankungen im einzelnen ist doch sämtlichen Erzählungen der eine Zug der Beteiligung des Menelaus gemeinsam und von 2 gegensätzlichen Ueberlieferungen kann nicht die Rede sein, zumal im Hinblick auf die sonstige im Wortlaut wie in der Anordnung herrschenden Uebereinstimmung. So erzählen die Byzantiner einschliefilich des Kedrenos völlig identisch: Helena wird von Priam und Hekuba bewillkommnet; letztere umarì und külst die Freunde.

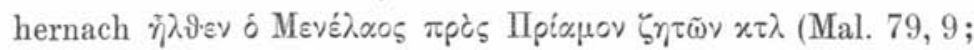
Kedren 218, 8 sqq). Genau entsprechend ist Septimius I 9 u. 10: Begrüfung: igitur Hecuba ... Helenam complexa ... deinde postero die Menelaus . . in contionem venit, coniugem

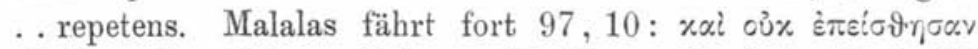

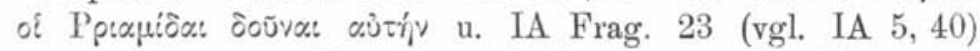

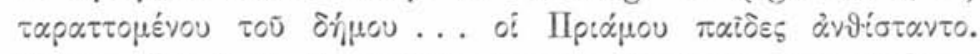
Septim. I 7 (p. 7, 4) erzählt: (wegen Rückgabe der Helena) cunctis indignantibus tumultus ortus est, während die Priamiden una voce minime reddendam Helenam respondent (vgl. auch Sept. I 18). - Eine Diskrepanz besteht zwischen Septimius und den Byzantinern auch hinsichtlich der zeitlichen Aufeinanderfolge der Gesandtschaft und der Ankunft der Helena in Troia. Die Byzantiner erwähnen zuerst das Eintreffen der Helena in Troia und dann lassen sie die Kunde davon nach Griechenland gelangen (Mal. 97, 3. Kedren 218, 18. IA 5,25 fr. 23) ${ }^{104}$ ); hierauf geht eine Gesandtschaft nach Troia ab. Im lateinischen Text hingegen werden alsbald nach

${ }^{104)}$ Manasses erzählt hier ganz summarisch. 
dem Ruchbarwerden des Raubes Gesandte nachgeschickt, die einige Tage vor Paris und Helena ankommen, Sept. I 5 (p. 5, 17). Wo haben wir die originale Erzählung zu suchen? Mit Sicherheit läft sich die Frage nicht entscheiden, soviel muf Noack zugestanden werden. A,ber eine Instanz von nicht zu unterschätzendem Gewicht spricht doch zu gunsten der Byzantiner.

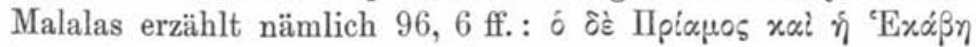

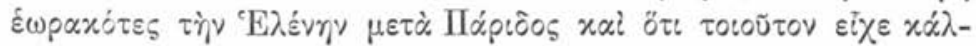

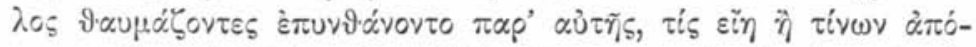

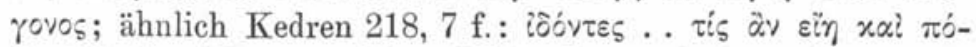

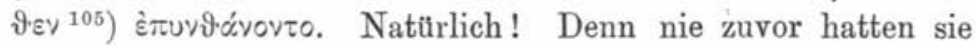
von Helena etwas gehört noch gesehen. Die Frage ist also ganz an ihrem Platze. Anders bei Septimius. Er hat dieselbe an einer Stelle (I 9), wo sie völlig unmotiviert und geradezu sinnlos ist. Denn Priamus, der ja die Frage stellt, und die reguli muften doch durch die vorher (I 6) erzählten Verhandlungen mit der Griechengesandtschaft über die persönlichen Verhältnisse der Helena genau unterrichtet sein. Septimius hat also wohl gedankenlos umgestellt, und die ursprünglichere Fassung dürften die Byzantiner bieten.

5. Die Begleitung des Achill nach Troia. Bei Septimius I 9 begleitet Phoenix den Achill auf dem Zug gegen Troia, bei Malalas 97, 22 aber Nestor (so auch Kedren $219,1{ }^{106}$ ). Auch Tzetzes Antehom. 185 (und wohl von ihm abhängig der auctor Uffenbachianus II 665, s. Greif S. 196) spricht von Phoenix. Die Begleitung des Achill durch Phoenix ist die homerische und die auch später gewöhnliche (vgl. Apollodor Mythogr. gr. 113, 15 West.). Wenn wir bei dem homerisierenden Tzetzes die homerische Version wiederfinden, so kann uns das nicht wundernehmen. Aus Homer hat wohl auch Septimius den Namen des Phoenix an Nestors Stelle ein-

$\left.{ }^{105}\right)$ Bei Septimius lautet die Frage: quae cuiusque esset. Das quae schließt eigentlich die Frage nach dem Vater, der Familie und sozialen Stellung notwendig ein, nicht aber die Frage nach der Landsmannschaft. Mir erschiene es daher passender, wenn wir cuiasque lesen dürften.

${ }^{106}$ ) Auch in der Hektorlysis spielt Nestor bei Mal. 124, 8, IA 7, $33 \mathrm{H}$ und Kedren 224, $10 \mathrm{ff}$. consequenterweise die Rolle, die von Septimius dem Phoenix zugeteilt ist. 
gesetzt (Sept. I $16=$ Il. IX 223). Dunger scheint diese ganze Differenz entgangen zu sein. Wie würde er daraus für seinen Standpunkt Kapital geschlagen haben!

6. Der Zug gegen die trois chen Nachbarsta a ten. Hier bietet bald Mal. 99, 13, bald Sept. II 13 ein Mehr. Greif $\$ 198$ hat mit Unrecht daran Anstof genommen. Die thatsächlichen Differenzen fallen sicherlich nur der Willkür der einzelnen Autoren zur Last. Das zeigt die folgende Stelle. Sept. p. 26, 5 heift es, man habe dem Aias die Glauke exceptam reliquae praedae habendam zugestanden. Diese Bemerkung wird erst voll verständlich durch Mal. 99, 15, wo die Griechen schwören, ö $\tau: ~ \pi \dot{\alpha} v \tau \alpha \tau \dot{\alpha} \pi \alpha \rho \dot{\alpha}$ ( $\tau \tilde{\omega} \nu$ $\pi \lambda \eta \eta \sigma i o v ~ \pi \dot{o}-$

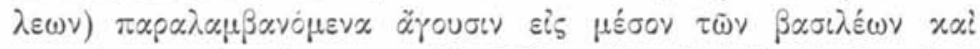

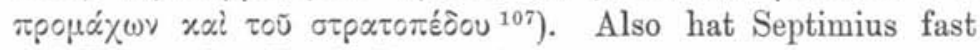
auf Kosten der Deutlichkeit gekürzt.

7. Der Schiffskatalog ist bereits erschöpfend abgehandelt von Haupt, Philolog. Anzeiger 1880 S. 541; Patzig, Byz. Zeitschr. I 146; Noack a. a. O. S. 462 ff. Einen Punct haben sie freilich übersehen. Manasses hat sicher denselben Schiffskatalog vor sich gehabt wie Mal. und Sept. Vergl.

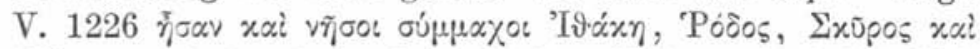

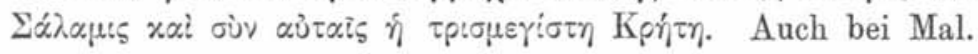
und Sept. stellt unter den Inseln Kreta das grölite Kontingent (= Il. II 645 sqq.). Auffallend aber ist, daf3 auch $\Sigma$ xúpos als

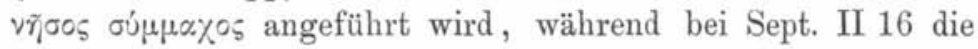
Stadt Skyrus auf der gleichnamigen Insel von Achill zerstört wird (= Il. IX 668). Auf seiten des Manasses ist wohl eine Erweiterung oder ein Mifverständnis anzunehmen.

8. Die Eroberung von Cyprus und Isauria. Mal. 102, 9 erzählt im Anschluj an den Bericht über die Beutezüge, dab Te ukros und Idomeneus Cyprus und Isauria erobert hätten. Septimius hat darüber nichts ${ }^{108}$ ). IA 7, 8 H erzählt allerdings das factum wie Mal.; nur ist es bei ihm Aias, der die Expedition unternimmt. Ein Versehen irgend welcher Art liegt nicht vor. Denn 6, 36 leitet IA den

$\left.{ }^{107}\right)$ Die Stelle beweist zugleich deutlich, daß Malalas jedenfalls nicht aus dem lateinischen Buche geschöpft hat.

$\left.{ }^{108}\right)$ Desgleichen Kedren und Manasses. 
Bericht über die Expeditionen mit der Bemerkung ein, die Griechen hätten, um sich im Rücken zu decken, beschlossen, die umliegenden Städte anzugreifen (= Sept. II 13) und damit den Aias, Achilleus und Diomedes beauftragt (= Mal. 99, 18 Ecloge 202,4). Möglich dafs aus ihr die Rolle des Aias bei IA abgeleitet ist. Keinesfalls aber scheint mir hier - tief im Diktysstoff! - eine Berufung auf den Einflufs des Sisyphos angebracht (Gleye, Byz. Zeitschr. V 455).

9. Die Homerika. Die in der Diktysüberlieferung aufstoßenden H. stammen gewißs aus dem griechischen Diktys und sind nicht erst aus Homer von den einzelnen Autoren eingefügt worden (vgl. darüber ausführlicher Greif S. 205 (§ 207); Noack S. 472). Einmal widersprechen sich die Byzantiner und Septimius. Patroklos' Fall und die Wiederbeteiligung Achilles am Kampfe erzählen die Byzantiner nach Homer (Kedr. 223, 14-20; Manasses 1343 sqq. IA 7,18 H vgl. Patzig, Byz. Zeitschr. IV 26. Körting S. 25), während bei Sept. sich Achill schon vor Patroklos' Tod mit den Griechen versöhnt und in den Kampf eintritt (II 51. III 5). Wer kommt hier der Diktysvorlage am nächsten? Noack S. 473 hat den "Eindruck", als ob Septimius die einfachere Form der Vorlage zu übertrumpfen suchte. Wer bürgt uns denn aber dafür, daßs die Vorlage die einfachere Form bieten mufte? Bedenkt man, daß Diktys Augenzeuge des Krieges sein will und alle diese Schwindler nicht selten von der homerischen Version abgingen, um unterrichteter zu scheinen, so wird klar, wie viel dieser "Eindruck" wert ist. Es ist durchaus ebenso denkbar, daf das griechische Original die septimianische Darstellung enthielt und vielmehr die Byzantiner es waren ${ }^{109}$ ), die nach Homer simplifizierten.

10. Die Ermordung des Palamedes. Dieses Stück bietet Schwierigkeiten, an denen man nicht vorübergehen darf. Die Byzantiner haben die seit der griechischen Tragödie geläufigste Version ${ }^{110}$ ). Kedr. 220,4 erzählt, Odys-

$\left.{ }^{109}\right)$ Malalas hat den ganzen Passus ubergangen, wie die genaustimmende Ecloge beweist. Die anderen Gewährsmänner (Kedr., Manass.) gehen wohl auf IA zurück.

i10) Noack S. 471.

Philologus LX (N. F. XIV), 3 . 
seus habe jenen mittels gefälschter Briefschaften des Hochverrates bezichtigt, weshalb Palamed gesteinigt, verbrannt und seine Asche an Nauplios gesandt worden sei. Bei Mal. hat davon nie etwas gestanden (s. o.) und auch in dem fragmentarischen IA vermifst man diese Erzählung. Septim. seinerseits hat eine bis auf ihn unerhörte ${ }^{111}$ ) Darstellung II, 15 : Odyseus und Diomed conspirieren gegen Palamed, locken ihn unter ziemlich plumpem Vorwand in eine Grube, wo sie ihn arreptis saxis, quae circum erant, tot werfen. Vergleichen wir sie mit der byzantinischen, so ergeben sich folgende gemeinsame Züge: a) die Ermordung geschieht mit auf Anstiften des Odysseus, b) Palamend wird gesteinigt, c) er wird tief betrauert (Manasses 1330 sqq. = Sept. p. 27, 17). Das ist aber der Kern der ganzen Erzählung. Greif ${ }^{112}$ ) und nach ihm Noack ${ }^{113}$ ) haben die nicht zu leugnenden Differenzen zu einem unausgleichbaren Gegensatz aufgebauscht. Es finden sich ja bei Sept. gewif Züge singulärer Art (der Schatz

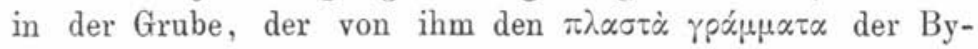
zantiner substituiert ist; die Steinigung durch Odysseus und Diomedes a llein; die Trauer des Achilles allein). Aber der Faden, an dem dieses eigenartige Beiwerk aufgereiht ist, ist bei Sept. kein anderer als bei den Byzantinern. Aber das ist die Hauptsache und genügt, um wenigstens die Möglichkeit offen zu halten, dafs beide Berichte aus ein und derselben Vorlage geflossen sind. Auf welcher Seite die umgemodelte Fassung liegt, ist natürlich nicht mehr zu sagen.

11. Das Hilfegesuch des Priam an David und Tautanes. Es wird überliefert von IA 7, 16; fragm. Salm. vgl. Mal. 127, 10 (Ecl. 219,6). Die Bitte an David ist natürlich christliche Zuthat. Daf aber auch das Gesuch an Tautanes auf einen alten Schriftsteller zurückgehen könne, haben Dunger ${ }^{114}$ ) und Sotiriades ${ }^{115}$ ) mit Unrecht geleugnet. Es steht schon Diodor II 22 und im armenischen Eusebius (FHG III 626 z. vgl. Niebuhr, Abhandlung. der Berliner Akademie d. W. $1820-21$ hist. phil. Kl. S. 37 ff. $\left.)^{116}\right)$. Wir müssen es für das griechische Diktysbuch reklamieren.

${ }^{111}$ ) Körting D. und D., S. 53.

112 S. $200 .^{113}$ ) S. $471^{114}$ ) S. $13 .{ }^{115}$ ) S. 24 A 19.

${ }^{116)}$ Noack S. 434 versteht unter Kephalion, auf den sich Euseb. 
12. Der Tod des Paris und der Oinone. Bei Mal. 111, 3 wird Paris von Philoktet mit den Heraklespfeilen tötlich verwundet und noch lebend zur Stadt gebracht, wo er in Gegenwart seiner von Helena erhaltenen Söhne stirbt. Oinone ${ }^{117}$ ) erhängt sich, als sie der Leiche ansichtig wird. So auch Kedr. 229, 3 und Tzetzes Posthom. 598 unter ausdrücklicher Berufung auf Diktys ${ }^{118}$ ). Bei Sept. IV 19. 20 dagegen erliegt Paris den vergifteten Geschossen des Philoktet s o fort, als Leiche bringt man ihn nach Troia. Bei deren Anblick stirbt Oinone vor Gram IV, 21. Von den Helenasöhnen spricht Sept. an dieser Stelle nicht, einige Seiten später zeigt er aber, dafs er ihre Namen kennt V. $5^{119}$ ). Die Stelle hat den Diktysforschern viel Kopfzerbrechen gemacht ${ }^{120}$ ). Man wird sich mit der Vermutung bescheiden müssen, daf3 wir es hier bei Septimins wirklich einmal mit einer Outrierung des ursprünglichen Diktystextes zu thun haben. Die Sucht, originell zu erzählen, mag den Uebersetzer verleitet haben, die gewöhnliche ${ }^{121}$ ) Version zu verlassen und ihre eine andere Pointe zu geben.

13. Achill und Polyxena. Sein Tod. Septimius' Erzählung hat die Voraussetzung, wie IV 11 p. 77,11 beweist, dafs der Tempel, in welchem Achill die Polyxena erblickt, mit jenem, in welchem die Verhandlungen über die

beruft, den Gergithier Keph. (Hegesianax), der Troica schrieb (Ath. $393^{\mathrm{d}}$ Christ, Gesch. der griech. Litter. S. 474). Neuestens hat E. Schwartz Abhandl. d. Akad. d. W. zu Göttingen XI 1894-95 S. 20 an Keph., den Schwindler aus der Zeit Hadrians gedacht (Christ S. 565. Müller THG III 626 sqq.).

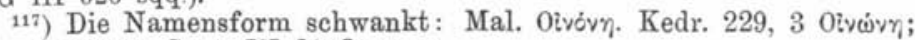
IA 9, 15 Oióvn; Sept. III 26 Oenone.

118) Ueber das gegenseitige Abhängigkeitsverhältnis vgl. Greif S. 220. Patzig Byz. Zeitschr. I, 139; Noack S. 474. 480.

${ }_{119}$ ) K. Lehrs, Populäre Aufsätze S. 16 sqq. ist geneigt, die Fabel

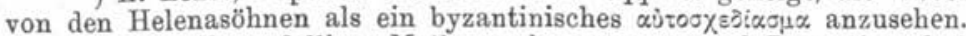
"Irgend ein selbstgefälliger Mythenverbesserer, zumal Byzantiner hat es anständig gefunden, daß dem kgl. Paar unter dem Sclutz der Aphrodite der Kindersegen nicht versagt geblieben." Von byzantinischer Erfindung kann nicht die Rede sein. Denn schon die alexandrinischen Grammatiker kannten dieses Motiv, vgl. Eust. Il. I 40: Dardanus; einen Coryttus nennt Nikander bei Parthenius 34 vgl. Roscher, Myth. Lex. s. v. Helena.

${ }^{120}$ ) Perizonius LXIII sq., Körting D. u. D. S. 42. Sein eigener Erklärungsversuch ist ein Muster von Textmißhandlung (S. 41).

${ }^{121}$ ) Perizonius LXIV. Körting S. 44. 
Vermählung und Ermordung des griechischen Helden stattfinden, identisch ist und außerhalb der Stadt liegt. So auch Mal. 130, 8 und 131, 12; IA 8, 37 H; Kedr. 227, 12; 228, 8 sq. z. vgl. Dares c. 34 . Bei Manasses ist die Sache dunkel. V 1382 spricht er von einem vor der Stadt gelegenen Tempel,

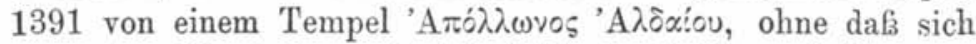
aus seiner Darstellung die Identität der beiden mit Notwendigkeit ergäbe. Denn 1396 ergreifen die Mörder die Flucht, $\mathrm{ob}$ in die Stadt, wird nicht gesagt. Ihm folgt die Vulgär$\operatorname{chronik}^{122}$ ). Wir werden diese unklare Stelle nach jener einstimmigen Ueberlieferung interpretieren dürfen.

$\mathrm{Ob}$ auch das bei sämtlichen Byzantinern vermifite Stück Sept. III 4 (die Verhandlungen Achills wegen Polyxena) dem Diktysstoff zuzurechnen sei, ist schwer zu sagen. Wenn Noack ${ }^{123}$ ) darauf hingewiesen hat, daf durch die Reklamierung jenes Abschnittes für das Diktysoriginal das Anerbieten des Priam (Mal. 125, 10 sqq. Sept. p. 69, 19; IA 7, 4 cfr. Manasses 1384) motivierter erscheine, so hat er übersehen, dafs bei Sept. l. c. nicht Priam, sondern Hector und zwar der Natur der Sache nach heimlich, mit dem Peliden verhandelt.

14. Das hölzerne Pferd. Helenus. „Bei Sept. V 9 giebt Helenus, bei Malalas 113, 5 Odysseus den Rat, das Pferd zu bauen ". So Noack S. 475. Bezüglich des Byzantiners ist das zu viel behauptet. Denn Mal. läßt den Odysseus (in der Palladionkrisis) nur sagen, daßs er die Griechen beschworen habe, nicht abzusegeln, bis sie das hölzerne Rof verfertigt und den Scheinrückzug nach Tenedos ausgeführt hätten. Er mift sich also nur das Verdienst bei, daf er die kampfesmüden Griechen abzufahren verhindert habe, bevor sie es mit jener Kriegslist versucht hätten ${ }^{124}$ ). Daß3 er auch ihr geistiger Urheber sei, sagt er nicht, so wenig als bei Kedren 230, 17. Vielmehr läßst der ganze Wortlaut (vgl. auch den Artikel bei ooúp:os ( $\pi \pi \circ \varsigma)$ durchblicken, daf die Idee, als Odysseus eingriff, bereits von einem anderen angeregt war.

$\left.{ }^{122}\right)$ Prächter, Byzant. Zeitschr. IV 545.

12s) S. 473. p. $16 \mathrm{sq}$.

${ }^{124}$ ) vgl. J. O. Schmidt, Ulixes Posthomericus part. I. Berlin 1885 


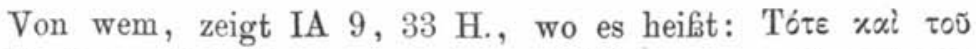

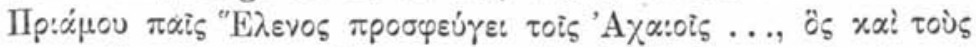

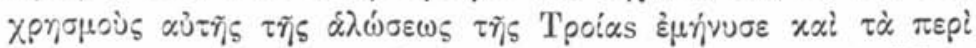

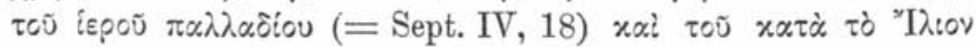

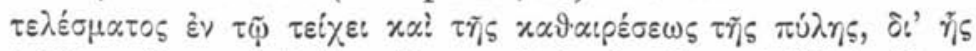

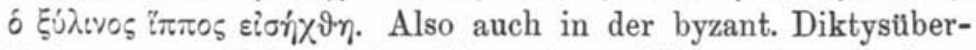
lieferung ist Helenus der Vater jener List und von einem Gegensatz kann also keine Rede sein.

15. Die Verstümmelung des Deiphobos. Sept. V, 12 p. 97,12 erzählt ausführlicher als die (meisten) Byzantiner, und Noack ${ }^{125}$ ) glaubt daher, daf er in seinem Detail dem Vergil (Aen. VI 494 ff.) gefolgt sei. Es ist aber zu betonen, dafs auch der neue IAtext 10,12 H Einzelheiten giebt: neben Verstümmelung der Hände auch Verstümmelung der Füke und die Bemerkung, dafs auch dessen Kinder hingeschlachtet wurden - was sich alles wie bei Sept. so auch bei Vergil nicht findet. Die Darstellung im griechischen Original kann also sehr ausführlich gewesen sein und von einigen dem Vergil entlehnten Verzierungen ${ }^{126}$ ) abgesehen aus ihm Sept. geschöpft haben.

16. D i e Tel e g o ni e. Kedr. 223, $13-22$ hat sie kurz zusammengefafit: weit ausführlicher steht sie beim Eclogarius $=$ Sept. VI, 15. Dunger, Dikt.-Sept. S. 28 hat sie dem Homer (!) in das Koffer packen wollen, aber schon Noack ${ }^{127}$ ) hat nicht ermangelt, ihm in gebührender Weise ein Lichtlein aufzustecken. Freilich von dem, was Ecl. 215, 32-216, 5 über die Teilung des Reiches des Odysseus zu lesen ist, steht bei Sept. nichts ${ }^{128}$ ). Gleichwohl hat der griechische Diktys ein Anrecht auf diesen Abschnitt, denn

a) der Bericht hängt mit der Telegonie eng zusammen

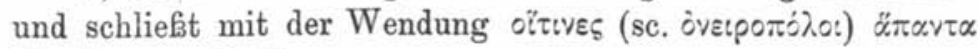

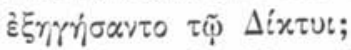

b) gerade hier wird Ptoliporthos, der Sohn des Telemach und der Nausikaa, genannt $=$ Sept. VI 6 .

$\left.{ }^{125}\right)$ S. 476.

$\left.{ }^{126}\right)$ Noack S. 456 bes. a. 63 ; 476 a. 84. Patzig, Byz. Zeitschr. IV 29 ; dagegen Greif S. 222.

$\left.{ }^{127}\right)$ S. 415 ; vgl. Lehrs a. a. O. S. 136 ; Fuchs p. 178. 183.

${ }^{128)}$ vgl. Patzig, Byz. Zeitschr. I 148. 
17. Äneas und Antenor. Septim. I 3 (p. 4, 16) und V 2 (p. 87,14) weiß, dak Aeneas mit Glaukos und andern Verwandten den Paris nach Sparta begleitet habe. Noack ${ }^{129}$ ) sagt - freilich ohne alle Begründung - dieser Zug könne wohl bei Diktys gestanden haben. Sicherlich!

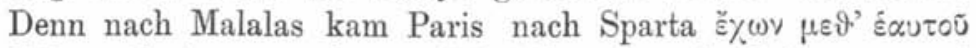

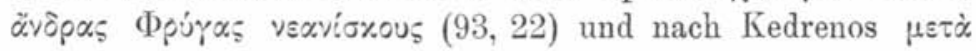

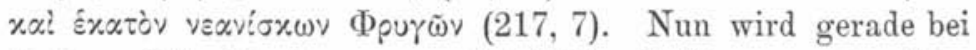
Kedren 245, 19 Aeneas ausdrücklich $\Phi$ púg genannt. Also hindert nichts, ihn jenem phrygischen Gefolge des Paris beizuzählen. Aber auch die übrigen auf Aeneas bezüglichen Notizen des Septimius können Eigentum des griechischen Originals gewesen sein. Ein Römer würde von sich aus schwerlich ein so zweideutiges Bild von seinem Nationalhelden gezeichnet haben, wie es uns thatsächlich in der Diktysüberlieferung entgegentritt ${ }^{130}$ ). Dungers Behauptung ${ }^{131}$ ), Aeneas sei von Septimius bevorzugt ${ }^{132}$ ), ist eine wahre Unbegreiflichkeit. Was wird ihm denn Rühmliches nachgesagt? Wenn er II 11 p. 24, 28 den Protesilaus tötet, so will ihm das, wie schon der Wortlaut erkennen lälist, gar nicht als besonderes Verdienst angerechnet werden, so wenig als jenem obscuren $\Delta$ apōavòs ¿̀vip, der bei Homer B 701 diese Heldenthat vollbringt. Dagegen erscheint Aeneas in recht ungünstigem Licht an verschiedenen Stellen. Er ist in der Gesellschaft des weiblichen

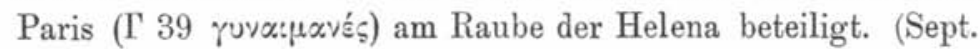
I $3=$ Mal. 93, 22). Sept. IV 22, V 1 . ist er in verräterische Umtriebe verwickelt und V 17 intriguiert er in perfider Weise gegen Antenor. Stammt diese Zeichnung aus einer griechischen Quelle, so hat sie nichts Anstößiges mehr.

Die Ecl. 221, 25 berichtet, Aeneas sei nach Westen ge-

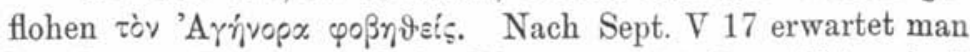
'Avтrívopo. Eine eigentliche Discrepanz ist das nicht vgl. $\Xi$

${ }^{129)}$ S. 466 a. 70.

${ }^{130}$ ) vgl. Hertzberg a. a. O. S. 179.

131) S. 33.

134) Bei Homer steht Aeneas weit höher als bei Sept. E 344 × 391 ist er der Liebling der Götter. II 620 wird seine Tapferkeit hervorgehoben. E 217; 227 wird er der tapferste Trojaner nach Hector genannt (vgl. $\Xi 425)$. 
$425 ; \Lambda 59$, wo Aeneas neben Agenor genannt ist. Vielleicht hat infolge dieser äuferiichen Verbindung der Namen ein ähnlicher Vorgang stattgefunden, wie er mitunter bei Verg. z. B. Aen. IX $176=\mathrm{I} 20$; X $123=\Delta 20$; X $123=$ T 146 $\mathrm{zu}$ constatieren ist $\left.{ }^{133}\right)$.

Schon oben habe ich darauf hingewiesen, dab die Verbindung des Antenor mit Aeneas eine charakteristische Eigentümlichkeit des lateinischen Textes sei. Wir werden sie aber schon für den griechischen Dictys voraussetzen müssen. Eine Spur hievon zeigt die oben citierte Stelle der Ecloge 221, 25, besonders aber das griechische Original der rumänischen Troica $^{134}$ ), wo cap. 38 die vertragsmäßige Verschwörung des Aeneas und Antenor fast wörtlich erzählt ist wie Sept. III 26. Sie findet sich bereits bei Livius I, 1 und ,ist eine der drei Fassungen, in denen die älteren römischen Darstellungen den Abzug des Aeneas erzählen ${ }^{135}$ ). In der griechischen Litteratur ist der älteste Zeuge für diese Verkoppelung ein gewisser Abas (Müller FHG IV 278), dessen Lebenszeit wohl vor Vergil und Livius zu setzen ist (vgl. Servius ad. Aen. IX 264).

Tübingen.

J. Fïrst.

$\left.{ }^{133}\right)$ Cauer Fleckeisens Jahrb. Suppl. XV S. 179 f.

134) Prächter, Byz. Zeitschr. IV 547.

135) Cauer a. a. O. S. 162; vgl. noch Fuchs 140. 